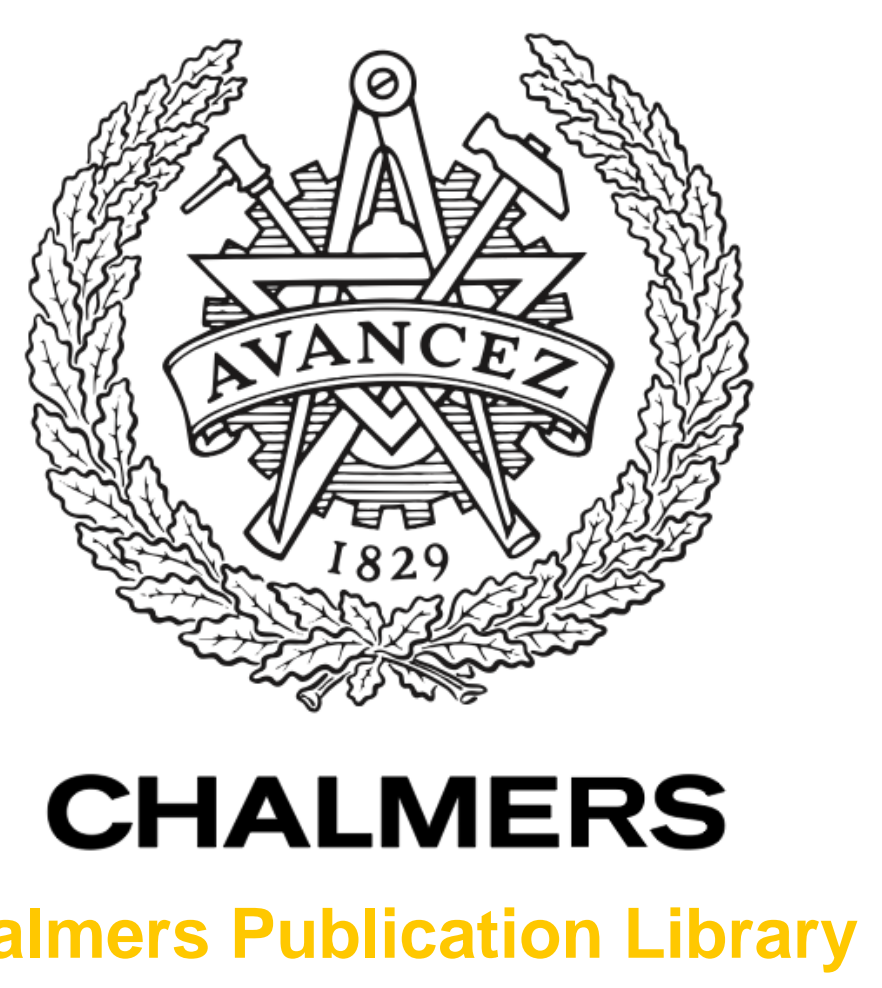

Chalmers Publication Library

\title{
Location-aided mm-wave channel estimation for vehicular communication
}

This document has been downloaded from Chalmers Publication Library (CPL). It is the author's version of a work that was accepted for publication in:

\section{IEEE 17th International Workshop on Signal Processing Advances in Wireless}

Communications (SPAWC), Edinburgh, England, 3-6 July, 2016 (ISSN: 2325-3789)

Citation for the published paper:

Garcia, N. ; Wymeersch, H. ; Ström, E. et al. (2016) "Location-aided mm-wave channel estimation for vehicular communication". 2016 IEEE 17th International Workshop on Signal Processing Advances in Wireless Communications (SPAWC), Edinburgh, England, 3-6 July, 2016

http://dx.doi.org/10.1109/SPAWC.2016.7536855

Downloaded from: http://publications.lib.chalmers.se/publication/240210

Notice: Changes introduced as a result of publishing processes such as copy-editing and formatting may not be reflected in this document. For a definitive version of this work, please refer to the published source. Please note that access to the published version might require a subscription. 


\title{
Location-Aided mm-Wave Channel Estimation for Vehicular Communication
}

\author{
Nil Garcia*, Henk Wymeersch*, Erik G. Ström*, and Dirk Slock ${ }^{\dagger}$ \\ *Department of Signals and Systems, Chalmers University of Technology, Sweden \\ ${ }^{\dagger}$ EURECOM Mobile Communications Department, Sophia Antipolis, France \\ email: nilg@chalmers.se
}

\begin{abstract}
Millimeter-wave (mm-wave) communication is a promising technology for next-generation wireless systems. One challenging application lies in the vehicular domain, where mm-wave should support ultra-fast and high-rate data exchanges among vehicles and between vehicles and infrastructure. To achieve ultra-fast initial access between nodes, we propose a location-aided beamforming strategy and analyze the resulting performance in terms of antenna gain and latency. We find that location information can significantly speed up initial access.
\end{abstract}

\section{INTRODUCTION}

Millimeter wave (mm-wave) communication is a candidate for becoming part of the next generation of mobile communication technology (also known as 5G). Its advantage lies on its capability to support multi-Gbps throughput. However, one of the major challenges for mm-wave communications is the poor link budget, as radio signals propagating in the mm-wave frequency band experience significant path loss, penetration and reflection loss [1]. On the other hand, an interesting feature in mm-wave systems is that the decrease in wavelength enables packing many antennas elements in a single array. Such large antennas arrays can compensate the high propagation loss through their high beamforming gain. In order to obtain the highest combined gain between the transmitter and a receiver, it is necessary that as a first step the two antennas are aligned. This is commonly achieved through a multi-stage channel estimation process [2]-[4].

A key application of $5 \mathrm{G}$ lies in the automotive industry [5], which is currently undergoing several technological transformations, as more and more vehicles are connected to the Internet and to each-other. Mm-wave could provide the high throughput required in vehicular networks for safety and awareness applications [3], as well as cooperative control. Such vehicular networks operate under more severe conditions than common cellular networks due to their high mobility, which greatly reduces the coherence time. In the context of mm-wave, achieving high beamforming is especially challenging, as the beamforming process must be completed in an extremely short time. Standard protocols, which rely on adaptive beamforming are well-suited for static nodes, but might be too slow for quick interactions.

In this paper, we propose a location-aided strategy, whereby prior location information allows us to speed up the adaptive channel estimation and beamforming. To the best of the authors' knowledge this is the first location-aware channel estimation technique for mm-wave. We quantify the performance in terms of SNR and latency.

\section{SignAl MODEL}

We consider communication between a base station (BS) and a vehicle, both equipped with uniform linear arrays (ULAs) of $N_{\mathrm{BS}}$ and $N_{\mathrm{v}}$ antennas, with half-wavelength spacing between adjacent antennas. If the BS uses the beamforming vector $\mathbf{f}_{n}$ to transmit a symbol $s \in \mathbb{C}$ with unit-energy $|s|^{2}=1$, and the vehicle employs a unit-norm measurement vector $\mathbf{w}_{m}$, the received signal is given by

$$
y_{m, n}=\mathbf{w}_{m}^{\mathrm{H}} \mathbf{H} \mathbf{f}_{n} s+\mathbf{w}_{m}^{\mathrm{H}} \mathbf{n}_{m, n},
$$

where the mm-wave channel is represented by the $N_{\mathrm{v}} \times N_{\mathrm{BS}}$ matrix $\mathbf{H}$ and $\mathbf{w}_{m}^{\mathrm{H}} \mathbf{n}_{m, n}$ is white Gaussian noise with variance $\sigma^{2}$. The channel is modeled as a pure narrow-band line of sight (LOS) channel [6]:

$$
\mathbf{H}=\alpha \mathbf{a}_{\mathrm{v}}(\theta) \mathbf{a}_{\mathrm{BS}}^{\mathrm{H}}(\phi),
$$

where $\alpha \in \mathbb{C}$ denotes the deterministic unknown channel gain, $\mathbf{a}_{\mathrm{v}}(\theta)$ represents the array response of the vehicle's ULA to the LOS angle-of-arrival (AOA) $\theta$ and $\mathbf{a}_{\mathrm{BS}}(\phi)$ represents the steering vector of the BS's ULA for the LOS angle-of-departure (AOD) $\phi$, given by

$$
\begin{aligned}
\mathbf{a}_{\mathrm{BS}}(\phi) & =\left[\begin{array}{llll}
1 & e^{j \pi \cos \phi} & \cdots & e^{j\left(N_{\mathrm{BS}}-1\right) \pi \cos \phi}
\end{array}\right]^{\mathrm{T}} \\
\mathbf{a}_{\mathrm{v}}(\theta) & =\left[\begin{array}{llll}
1 & e^{j \pi \cos \theta} & \cdots & e^{j\left(N_{\mathrm{v}}-1\right) \pi \cos \theta}
\end{array}\right]^{\mathrm{T}} .
\end{aligned}
$$

Our goal is to determine $\mathbf{H}$ in order to utilize high-gain beamforming and measurement vectors, in the presence of location information of the vehicle.

\section{AdAptive Channel Estimation}

\section{A. Procedure}

Typically, in mm-wave, the channel is estimated by using all different combinations of $L$ beamforming vectors and $L$ measurement vectors in different time slots [2]-[4], resulting in a total of $L \times L$ measurements. Organizing the measurements in matrix form, we can write

$$
\mathbf{Y}=\mathbf{W}^{\mathrm{H}} \mathbf{H F} s+\mathbf{N},
$$

where $\mathbf{W}=\left[\mathbf{w}_{1} \cdots \mathbf{w}_{L}\right]$, and $\mathbf{F}=\left[\mathbf{f}_{1} \cdots \mathbf{f}_{L}\right]$ and $\mathbf{N} \sim \mathcal{C N}\left(0, \sigma^{2} \mathbf{I}\right)$. The statistical independence among the entries of $\mathbf{N}$ follows from the fact that they represent 


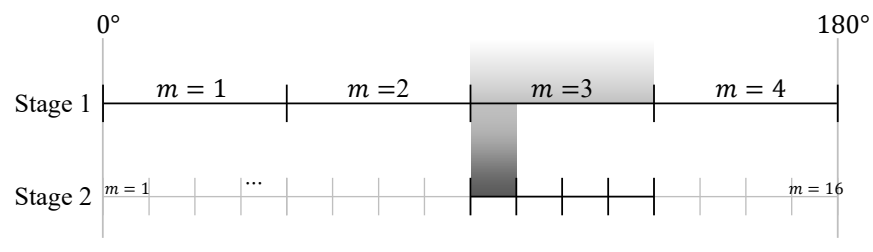

Fig. 1. Example of the adaptive channel estimation process at the receiver with $K=2$ stages and $L=4$ vectors per stage. The ranges of the beampatterns are designed according (10).

the noise at different time slots. We focus on the case of channel estimation techniques whose sets of weight vectors (at the transmitter and receiver) are precomputed off-line. Adaptive approaches refine the process of channel estimation in multiple stages. Hence, the signal model at stage $k$ is

$$
\mathbf{Y}_{(k)}=\mathbf{W}_{(k)}^{\mathrm{H}} \mathbf{H} \mathbf{F}_{(k)} s+\mathbf{N}_{(k)},
$$

where

$$
\begin{aligned}
\mathbf{W}_{(k)} & =\left[\begin{array}{lll}
\mathbf{w}_{\left(\hat{m}_{(k-1)}-1\right) L+1} & \cdots & \mathbf{w}_{\hat{m}_{(k-1)} L}
\end{array}\right] \\
\mathbf{F}_{(k)} & =\left[\begin{array}{lll}
\mathbf{f}_{\left(\hat{n}_{(k-1)}-1\right) L+1} & \cdots & \mathbf{f}_{\hat{n}_{(k-1)}}
\end{array}\right]
\end{aligned}
$$

in which $\hat{m}_{(k-1)}$ and $\hat{n}_{(k-1)}$ are the indices of the weight vectors selected in the previous stage $k-1$. The general idea, as portrayed in Fig. 1, is that at stage $k=1$, it is assumed that the AOD and AOA can be anywhere in the interval $[0, \pi)$ in the case of a ULA or $[0,2 \pi)$ for arbitrary arrays. Then, the transmitter and receiver sound the channel in multiple ranges of AODs and AOAs and the ones resulting in the strongest receive SNR are selected. In the subsequent stage, the previous ranges are subdivided into $L$ subranges. This process is repeated until the desired resolution is achieved. Thus, at stage $k$, there are $L^{k}$ possible beamforming and measurement weight vectors to choose from, which represent $L^{k}$ disjoint ranges at the BS and vehicle. Ideally, for well designed codebooks, the ranges associated to the weight vectors correspond to directions in which they have larger gains. The gain as a function of the AOD or AOA can be computed by

$$
\begin{aligned}
g_{\mathrm{BS}}(\phi) & =\left|\mathbf{a}_{\mathrm{BS}}^{\mathrm{H}}(\phi) \mathbf{f}_{n}^{(k)}\right| \\
g_{\mathrm{v}}(\theta) & =\left|\mathbf{a}_{\mathrm{v}}^{\mathrm{H}}(\theta) \mathbf{w}_{m}^{(k)}\right|,
\end{aligned}
$$

where the dependency of $g_{\mathrm{BS}}(\phi)$ and $g_{\mathrm{v}}(\theta)$ on indices $n, m$, and $k$ has been omitted to avoid overloading the notation.

\section{B. Codebook}

Different codebooks will result in different beampatterns, which in turn will result in different gains in different directions (9) (see [7], [8] for some codebook examples). For this work, we build on the method proposed in [2]. In the case of a ULA, there are two viable ways to design the beamforming and measurements weight vectors. Each beamforming/measurement weight vector is associated to a range of AOD/AOA angles. Let $\mathcal{R}_{m}^{(k)}$ be the range of the $m$-th weight vector at stage $k$ at the transmitter or receiver. On one hand, the weight vectors can be designed to have

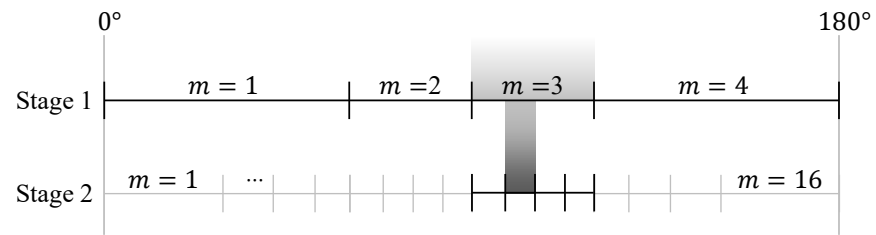

Fig. 2. Example of the adaptive channel estimation process at the receiver with $K=2$ stages and $L=4$ vectors per stage. The ranges of the beampatterns are designed according (11).
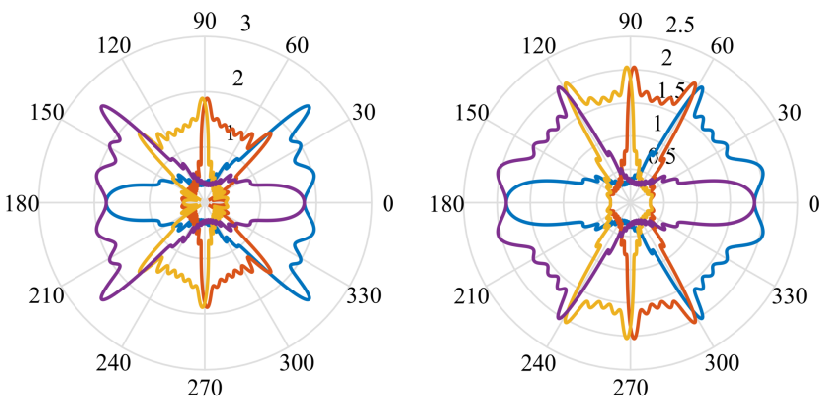

(a) Beampatterns designed (b) Beampatterns designed according to the ranges in (10). according to the ranges in (11).

Fig. 3. Beampatterns at stage 1 for $L=4$.

equal beamwidths at each stage as in Fig. 1, i.e. at stage $k$ the range of angles $[0, \pi)$ is split into $L^{k}$ subranges:

$$
\mathcal{R}_{m}^{(k)}=\left[\frac{m-1}{L^{k}} \pi, \frac{m}{L^{k}} \pi\right] \quad \text { for } m=1, \ldots, L^{k} .
$$

On the other hand, the weight vectors can be designed to have equal widths in terms of spatial frequencies (see Fig. 2):

$$
\begin{array}{r}
\mathcal{R}_{m}^{(k)}=\left[\arccos \left(1-\frac{m-1}{L^{k}} 2\right), \arccos \left(1-\frac{m}{L^{k}} 2\right)\right] \\
\text { for } m=1, \ldots, L^{k},
\end{array}
$$

where $0 \leq \arccos (x) \leq \pi$. Designing the codebook according to the ranges in (10) results in beampatterns of approximately equal beamwidth but unequal gain (see Fig. 3a). Designing the codebook according to the ranges in (11) results in beampatterns of approximately equal gains but whose beamwidths are wider on the endfire and narrower on the broadside of the ULA (see Fig. 3b). In this paper we choose the codebook designed according to the ranges of (11). The algorithm for designing the weight vectors $\mathbf{W}_{(k)}$ and $\mathbf{F}_{(k)}$ from the ranges $\mathcal{R}_{m}^{(k)}$ can be found in [2].

\section{Performance Metrics}

The metrics of choice for assessing the adaptive channel estimation techniques are:

1) Expected SNR: The expected receive SNR after performing adaptive channel estimation.

2) Average duration: The time it takes to perform adaptive channel estimation. ${ }^{1}$

\footnotetext{
${ }^{1}$ The time of a single transmission (1) is equal to 1 time unit.
} 


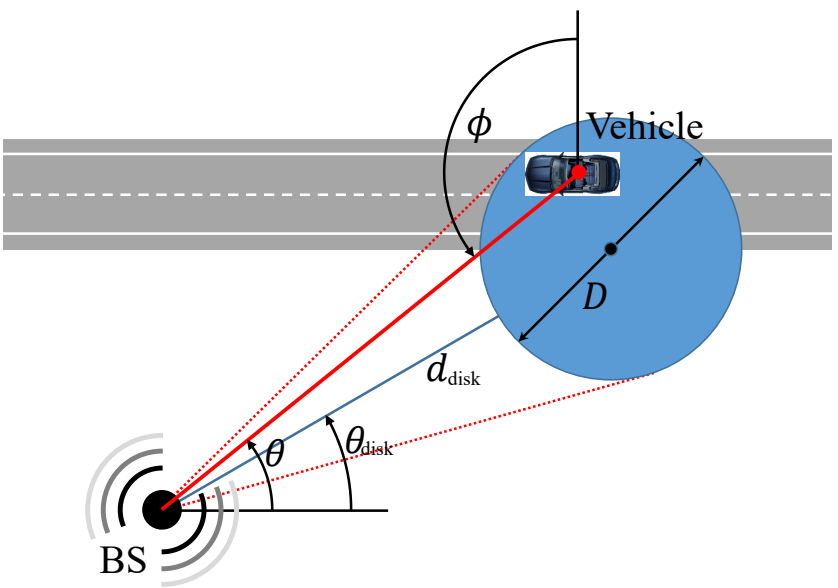

Fig. 4. Use case of interest, where prior information of the vehicle (represented by the blue disc) is harnessed to improve adaptive mm-wave channel estimation. In this particular scenario, the prior AOD and AOA prior information is $\mathcal{U}_{\theta}=\left[\theta_{\text {disk }}-\arcsin \left(\frac{D}{2 d_{\text {disk }}}\right), \theta_{\text {disk }}+\arcsin \left(\frac{D}{2 d_{\text {disk }}}\right)\right]$ and $\mathcal{U}_{\phi}=\left[\pi-\theta_{\text {disk }}-\arcsin \left(\frac{D}{2 d_{\text {disk }}}\right), \pi-\theta_{\text {disk }}+\arcsin \left(\frac{D}{2 d_{\text {disk }}}\right)\right]$, respectively, and $-\frac{\pi}{2} \leq \arcsin (x)<\frac{\pi}{2}$.

The parameters under control in adaptive channel estimation are the number of refinement stages $K$, the number of beams $L$ used at each stage by the BS and vehicle, and the codebooks at the transmitter and receiver.

\section{PERFORMANCE}

\section{IN THE PRESENCE OF POSITION INFORMATION}

It is our hypothesis that adaptive channel estimation can be improved in terms of the above performance metrics, when prior position information of the vehicle is available [9], as visualized in Fig. 4. In particular, we will assume that the vehicle's position is known to lie in a disk with radius $D$, with a center that is a distance $d_{\text {disk }}$ away from the BS, with an AOD of $\theta_{\text {disk }}$. This location information can be converted to information on the AOD/AOA by using simple trigonometry. Prior AOD/AOA information can then be utilized to discard AOD and AOA ranges that do not include the true AOD and AOA, respectively. This technique exploits the fact that given the BS and vehicle positions, the AOD/AOA of the LOS path can be inferred. Thus, it is likely to fail if the LOS path is blocked. With this clear adaptive channel strategy in mind, we can determine the effect of prior information using the various metrics of Section III-C. Formulas for these metrics are derived next.

Define the prior AOD/AOA knowledge as $\phi \in \mathcal{U}_{\phi}$ and $\theta \in \mathcal{U}_{\theta}$, where the sets $\mathcal{U}_{\phi}, \mathcal{U}_{\theta} \subseteq[0, \pi)$. Let $m_{(1)}, \ldots, m_{(K)}$ and $n_{(1)}, \ldots, n_{(K)}$ be the sequences of beamforming and measurement vectors indices, and let $P\left(m_{(K)}, n_{(K)}\right)$ be the probability that at the end of the channel estimation procedure the pair of weight vectors $\left(m_{(K)}, n_{(K)}\right)$ is picked when $s, \alpha$, $\theta$ and $\phi$ are fixed. Then,

$$
P\left(m_{(K)}, n_{(K)}\right)=\prod_{k=1}^{K} P_{(k)}\left(m_{(k)}, n_{(k)}\right)
$$

where $P_{(k)}\left(m_{(k)}, n_{(k)}\right)$ is the probability of choosing the pair of weight vectors $\left(m_{(k)}, n_{(k)}\right)$ at stage $k$. Statistical independence between stages follows from the fact that different stages happen in different time intervals. A pair of weight vectors is picked only if their received signal strength is the largest at that stage. Given $\left(m_{(k)}, n_{(k)}\right)$, from Fig. 1, it follows that at stage $k$, the indices of all the candidate beamforming and measurement weight vectors are

$$
\begin{aligned}
& m \in\left\{\left\lfloor\frac{m_{(k)}-1}{L}\right\rfloor L+1, \ldots,\left\lfloor\frac{m_{(k)}-1}{L}\right\rfloor L+L\right\} \\
& n \in\left\{\left\lfloor\frac{n_{(k)}-1}{L}\right\rfloor L+1, \ldots,\left\lfloor\frac{n_{(k)}-1}{L}\right\rfloor L+L\right\} .
\end{aligned}
$$

Therefore,

$$
P_{(k)}\left(m_{(k)}, n_{(k)}\right)=\operatorname{Prob}\left(\bigcap_{(m, n) \in \mathcal{S}_{(k)}}\left\{\left|y_{m_{(k)}, n_{(k)}}^{(k)}\right|^{2}>\left|y_{m, n}^{(k)}\right|^{2}\right\}\right),
$$

where $\mathcal{S}_{(k)}=\left\{(m, n):(m, n) \neq\left(m_{(k)}, n_{(k)}\right), \mathcal{U}_{\theta} \cap \mathcal{R}_{m}^{(k)} \neq\right.$ $\left.\emptyset, \mathcal{U}_{\phi} \cap \mathcal{R}_{n}^{(k)} \neq \emptyset\right\}$ and $\mathcal{R}_{m}^{(k)}$ and $\mathcal{R}_{n}^{(k)}$ represent the ranges of the beamforming and measurement vectors, respectively, defined as in (10) or (11) depending on the codebook design. By Bayes' theorem

$$
\begin{aligned}
& P_{(k)}\left(m_{(k)}, n_{(k)}\right)= \\
& =\int_{0}^{\infty} \operatorname{Prob}\left(\bigcap_{(m, n) \in \mathcal{S}_{(k)}}\left\{\left|\bar{y}_{m, n}^{(k)}\right|^{2}<x\right\} \mid x\right) f(x) \mathrm{d} x,
\end{aligned}
$$

where $\left|\bar{y}_{m, n}^{(k)}\right|^{2}=\left|y_{m, n}^{(k)}\right|^{2} /\left(\sigma^{2} / 2\right)$ is a noncentral Chi-square random variable of two degrees of freedom with non-centrality parameter

$$
\lambda_{m, n}^{(k)}=\frac{|\alpha|^{2} g_{\mathrm{BS}}^{2}(\phi) g_{\mathrm{v}}^{2}(\theta)}{\sigma^{2} / 2}
$$

and $f(x)$ is the probability density function of $\left|\bar{y}_{m_{(k)}, n_{(k)}}^{(k)}\right|^{2}$. Recall that the dependency of $g_{\mathrm{BS}}(\phi)$ and $g_{\mathrm{v}}(\theta)$ on $n, m$, and $k$ has been omitted. Since all variables $\bar{y}_{m, n}^{(k)}$ are mutually independent it follows that

$$
\begin{aligned}
& P_{(k)}\left(m_{(k)}, n_{(k)}\right)= \\
& \quad \int_{0}^{\infty} \prod_{(m, n) \in \mathcal{S}_{(k)}}\left(1-\mathrm{Q}_{1}\left(\sqrt{\lambda_{m, n}^{(k)}}, \sqrt{x}\right)\right) f(x) \mathrm{d} x,
\end{aligned}
$$

where $\mathrm{Q}_{1}\left(\sqrt{\lambda_{m, n}^{(k)}}, \sqrt{x}\right)$ is the complementary cumulative density function of $\left|\bar{y}_{m, n}^{(k)}\right|^{2}$ and $\mathrm{Q}_{1}$ is the Marcum Q-function of first order.

Expressions (12) and (18) can be used to numerically compute the expected receive $\operatorname{SNR} \operatorname{SNR}_{\mathrm{rx}}(\theta, \phi)$ and the average channel estimation duration $T(\theta, \phi)$ :

$$
\begin{aligned}
\operatorname{SNR}_{\mathrm{rx}}(\theta, \phi) & =\sum_{m=1}^{L^{K}} \sum_{n=1}^{L^{K}} P(m, n) \frac{g_{\mathrm{v}}^{2}(\theta)\left|\alpha^{2}\right| g_{\mathrm{BS}}^{2}(\phi)}{\sigma^{2}} \\
T(\theta, \phi) & =\sum_{m=1}^{L^{K}} \sum_{n=1}^{L^{K}} P(m, n) T_{m, n},
\end{aligned}
$$


where the dependency of $P(m, n)$ on $(\theta, \phi)$ is not written explicitly, $T_{m, n}=\max \left(\sum_{k=1}^{K}\left|\mathcal{S}_{(k)}\right|, 1\right)$ is the duration of the channel estimation procedure and $|\cdot|$ is the cardinality of a set. Even if the AOD/AOA is perfectly known a priori (i.e., $\sum_{k=1}^{K}\left|\mathcal{S}_{(k)}\right|=0$ ), at least one transmisson is necessary for estimating the channel gain.

\section{Numerical Results}

In this section, we quantify the performance of adaptive channel estimation when location information is available to the system.

\section{A. Simulation Set-up}

We consider the scenario of Fig. 4 versus the distance between the BS and the vehicle for two AODs: $20^{\circ}$ and $70^{\circ}$. These two angles have been selected as their performance is similar to all other angles. The scenario consists of a BS (infrastructure) with known position, and a vehicle whose position is uncertain. Different levels of precision are assumed in regards to the vehicle position estimate. This position uncertainty is modeled as a disk whose center point is known and whose diameter $D$ depends on the positioning technology. The position of the vehicle is known to be inside such a disk. In practice, the disk could correspond to a confidence region. We consider three levels of position information: (i) no position information $(D=+\infty)$, (ii) position information with Global Navigation Satellite Systems (GNSS) precision $(D=10 \mathrm{~m})$; and high precision position information $(D=1 \mathrm{~m})$.The simulations showed no substantial improvement for precisions higher than $1 \mathrm{~m}$.

From Fig. 4, given a fixed AOD, the AOA is fixed to $\theta=\pi-\phi$. As the distance increases, the SNR decreases due to the larger pathloss, however, it also narrows the prior AOD range $\mathcal{U}_{\phi}$ and prior AOA range $\mathcal{U}_{\theta}$. Thus, it is not clear how performance is affected by distance when location information is available. In the numerical simulations, we have assumed that the transmitted energy per symbol is $30 \mathrm{dBm}$, the vehicle's receiver's noise variance is $-84 \mathrm{dBm}$ and the path loss model of [6]. The figures in this section are Monte Carlo simulations where the average is taken over different disk locations while the position of the BS and vehicle remain fixed. The center of the uncertainty disk is randomly positioned on a circle of diameter $D$ around the vehicle. The BS is equipped with a ULA of 20 antennas and 9 radio-frequency $(\mathrm{RF})$ chains $^{2}$, and the vehicle has a ULA of 5 antennas and a single RF chain. The parameters of the channel estimation procedure are $K=2$ and $L=4$.

\section{B. Results and Discussion}

In Fig. 5, we plot the average channel estimation time versus the distance. As expected from the proposed strategy, in absence of prior information, the channel estimation lasts the longest and does not vary with the distance. In fact, it can be computed by the formula $T=K\left(L^{2}-1\right)$. Including

\footnotetext{
${ }^{2} \mathrm{~A}$ RF chain is composed of analog quantized phase shifters and up/down frequency converters. For more details see [2].
}

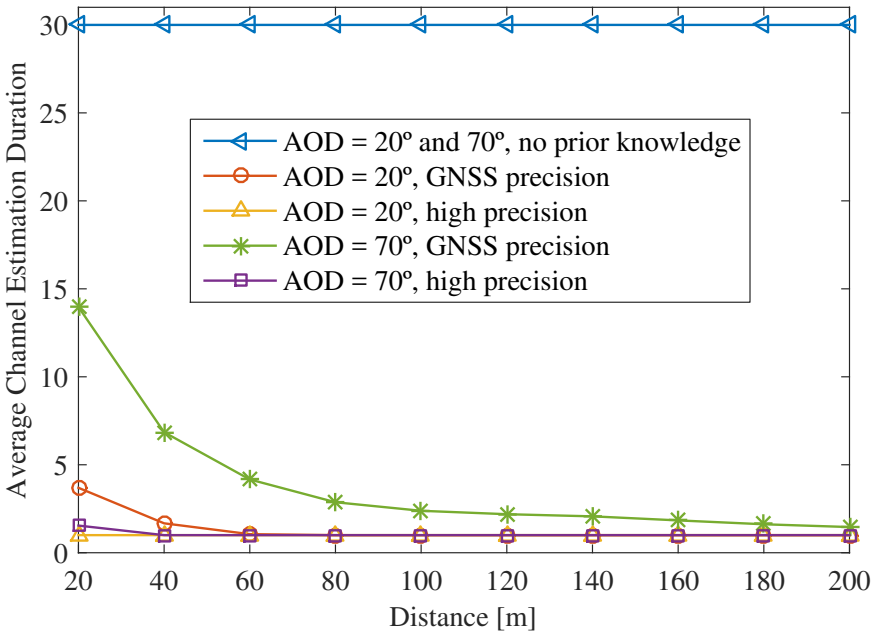

Fig. 5. Average channel estimation time versus distance. The time time axis is graded in "symbol durations".

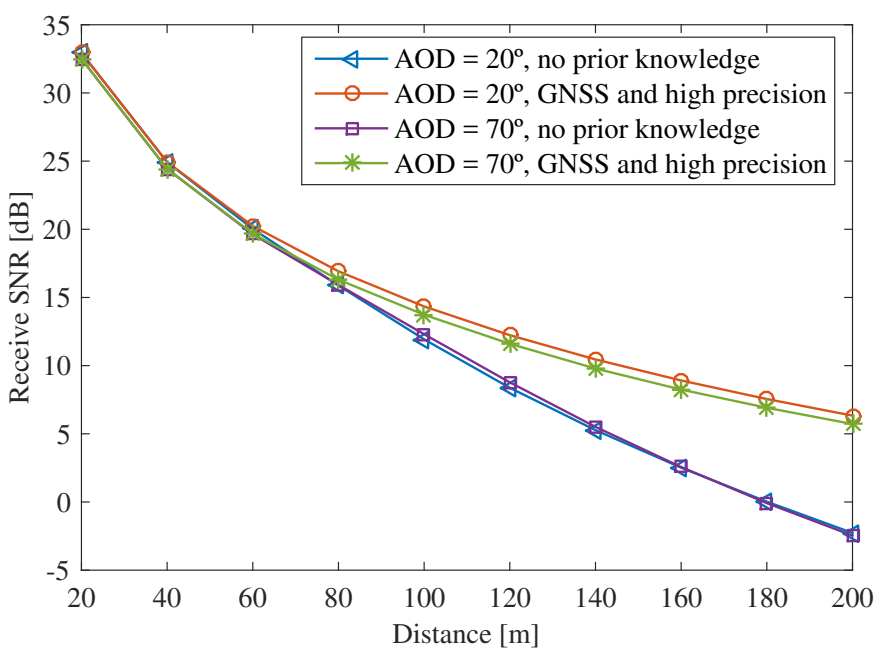

Fig. 6. Expected receive SNR versus distance.

coarse location information (GNSS) drastically reduces the channel estimation duration. Such reduction is larger as the distance increases because the ranges of AODs/AOAs become narrower, thus, reducing the number of directions on which the channel is sounded. In the case of high precision, the prior knowledge on the AOD and AOA is so precise to start with, that the location-aided channel estimation can already output the final weight vectors, i.e., no channel estimation is needed. In general, as observed in Fig. 5, the duration of the channel estimation is shorter for angles close to the endfire because the beams are wider (see Fig. 3b), and therefore, the channel needs to be sounded in fewer directions.

Fig. 6 plots the expected receive SNR after performing channel estimation versus distance. The behavior is almost identical for AODs $20^{\circ}, 70^{\circ}$ and other tested angles not plotted in the figure. When the vehicle is in proximity of the BS, the received power is large enough that location-aided and off-theshell channel estimation make no difference in terms of final receive SNR. As the distance increases beyond $80 \mathrm{~m}$, the use of 


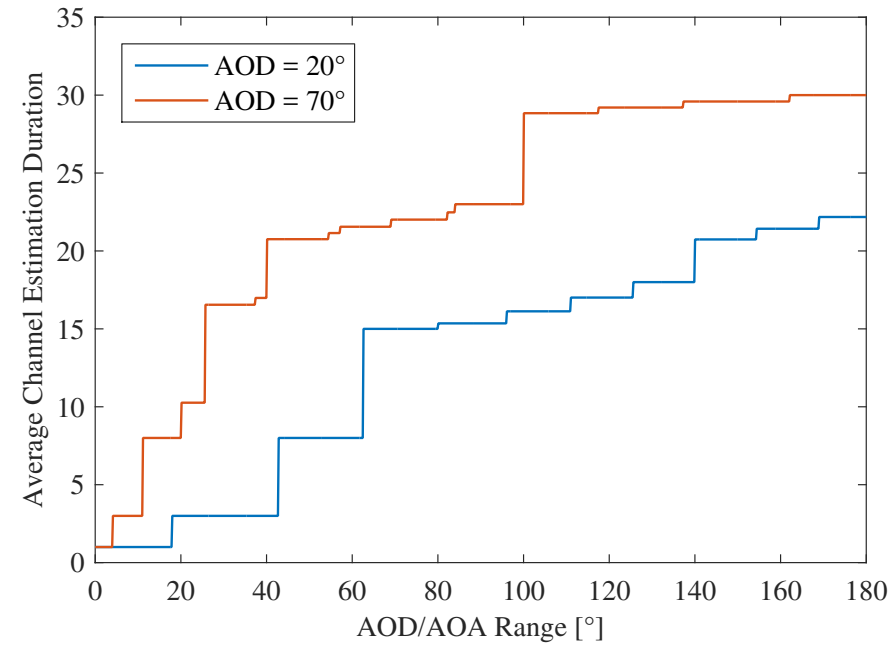

Fig. 7. Channel estimation duration vs. prior AOD/AOA range information. Distance between the BS and vehicle is fixed to $100 \mathrm{~m}$. The time time axis is graded in "symbol durations".

prior location information starts to become relevant because, by assisting the channel estimation procedure, it decreases the probability of misalignment. Interestingly, there's no significant difference between coarse (GNSS) or high precision location information. This phenomenon is due to the fact that the ranges of AODs/AOAs defined by the location uncertainty at $80 \mathrm{~m}$ for GNSS and high precision are equal or smaller than the width of the smallest beam $\left(\sim 7^{\circ}\right)$. In the case of massive ULAs, very directional beams may be possible, and therefore, precise location information may show significant improvement over coarse location information in terms of receive SNR. Nonetheless, it is challenging to use very narrow beams in practice, because they require that the antennas remain completely static for correct beam alignment which is very challenging in the V2I scenario and in the presence of wind [4].

For Figs. 7-8, the distance between the BS and the vehicle is fixed to $100 \mathrm{~m}$. The uncertainty disk is centered on the vehicle and its diameter is gradually changed, resulting in prior AOD/AOA ranges from 0 to $180^{\circ}$. As expected the duration and received SNR get worse for larger prior AOD/AOA ranges. The shape of both plots is due to the nature of the proposed offline approach, which sounds the channel with all transmit or receive beams whose ranges (11) overlap with the prior AOD range $\mathcal{U}_{\phi}$ or AOA range $\mathcal{U}_{\theta}$, respectively. The abrupt increase in duration or receive SNR occur when by increasing the prior AOD or AOA range, these ranges suddenly overlap with a new transmit or receive beam range. Thus, online approaches, whereby the ranges of the beams are adapted to the specific prior AOD or AOA range may result in better performance.

\section{CONCLUSIONS}

A simple adaptive channel estimation strategy for harnessing location information has been proposed. This strategy has been shown to allow the channel estimation time to be reduced substantially. Furthermore, numerical experiments have revealed that the receive SNR improves when location

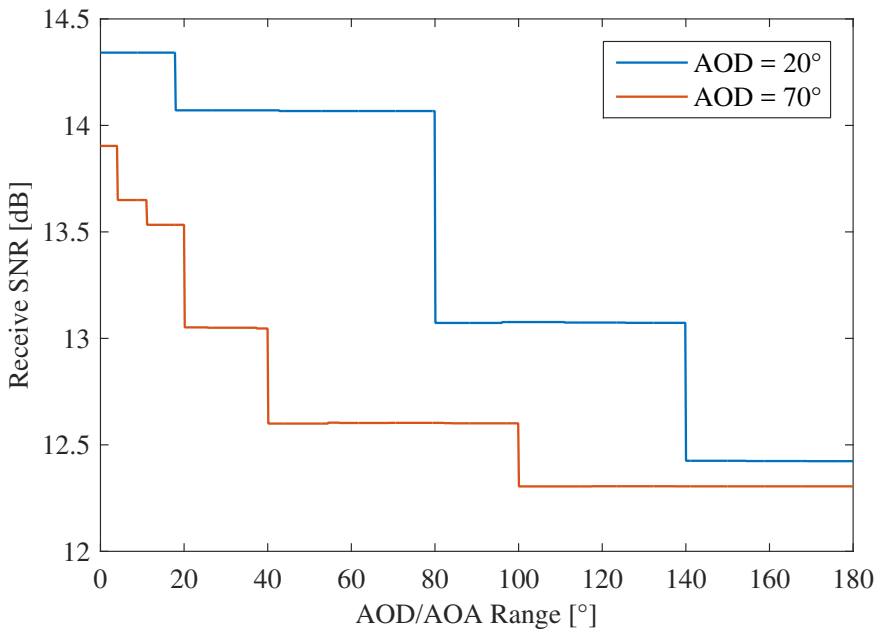

Fig. 8. Received SNR vs. prior AOD/AOA range information. Distance between the BS and vehicle is fixed to $100 \mathrm{~m}$.

information is available over distances of $80 \mathrm{~m}$ or more. Finally, our analysis has revealed that off-line approaches may have sub-optimal performance compared to online approaches especially in the presence of location information.

\section{ACKNOWLEDGMENT}

This research was supported in part, by the European Research Council, under Grant No. 258418 (COOPNET), and the EU HIGHTS project (High precision positioning for cooperative ITS applications) MG-3.5a-2014-636537.

\section{REFERENCES}

[1] T. S. Rappaport, S. Sun, R. Mayzus, H. Zhao, Y. Azar, K. Wang, G. N. Wong, J. K. Schulz, M. Samimi, and F. Gutierrez, "Millimeter wave mobile communications for 5G cellular: It will work!" IEEE Access, vol. 1, pp. 335-349, 2013.

[2] A. Alkhateeb, O. El Ayach, G. Leus, and R. W. Heath, "Channel estimation and hybrid precoding for millimeter wave cellular systems," IEEE Journal of Selected Topics in Signal Processing, vol. 8, no. 5, pp. 831-846, 2014

[3] J. Wang, Z. Lan, C.-W. Pyo, T. Baykas, C.-S. Sum, M. A. Rahman, J. Gao, R. Funada, F. Kojima, H. Harada et al., "Beam codebook based beamforming protocol for multi-gbps millimeter-wave WPAN systems," IEEE Journal on Selected Areas in Communications, vol. 27, no. 8, pp. 1390-1399, 2009.

[4] S. Hur, T. Kim, D. J. Love, J. V. Krogmeier, T. A. Thomas, and A. Ghosh, "Millimeter wave beamforming for wireless backhaul and access in small cell networks," IEEE Transactions on Communications, vol. 61, no. 10, pp. 4391-4403, 2013.

[5] ERTICO - ITS EUROPE, European Commission, 5G-PPP, "5G automotive vision," 2015.

[6] E. Ben-Dor, T. S. Rappaport, Y. Qiao, and S. J. Lauffenburger, "Millimeter-wave $60 \mathrm{GHz}$ outdoor and vehicle AOA propagation measurements using a broadband channel sounder," in GLOBECOM 2011. IEEE, pp. 1-6.

[7] Z. Xiao, T. He, P. Xia, and X.-G. Xia, "Hierarchical codebook design for beamforming training in millimeter-wave communication," IEEE Transactions on Wireless Communications, vol. 15, no. 5, pp. 3380-3392, 2016.

[8] A. Alkhateeb, O. El Ayach, G. Leus, and R. W. Heath, "Hybrid precoding for millimeter wave cellular systems with partial channel knowledge," in IEEE Information Theory and Applications Workshop, 2013, pp. 1-5.

[9] R. Di Taranto, S. Muppirisetty, R. Raulefs, D. Slock, T. Svensson, and H. Wymeersch, "Location-aware communications for 5G networks: How location information can improve scalability, latency, and robustness of 5G,” IEEE Signal Processing Magazine, vol. 31, no. 6, pp. 102-112, 2014. 